\title{
A cavernous hemangioma located in the axillary area: Challenges in preoperative diagnosis and operation
}

\author{
Jihye Choi', Chan Sub Park², Joonseog Kong ${ }^{3}$, Hyun-Ah Kim², Woo Chul Noh², Min-Ki Seong ${ }^{2}$ \\ 'Department of Surgery, National Medical Center, Seoul; \\ Departments of ${ }^{2}$ Surgery and ${ }^{3}$ Pathology, Korea Cancer Center Hospital, Korea Institute of Radiological and Medical Sciences, Seoul, Korea
}

Cavernous hemangiomas are benign neoplasms of endothelial cells. Although this neoplasm has the potential to develop in all parts of the body, it rarely develops in the axilla; in fact, there are only two case reports of axillary cavernous hemangiomas in the literature. Here, we describe a third case, which occurred in a 30-year-old Korean woman. The patient presented with a palpable mass in the left axilla that was initially thought to be either a phyllodes tumor or a lymphoma based on imaging studies. However, the results of an excisional biopsy led to a diagnosis of cavernous hemangioma. Although uncommon, a cavernous hemangioma can be encountered unexpectedly, presenting as a mass in axilla. Although usually curative, surgery may be challenging not only because of the rarity of the condition, but also because of inconsistent preoperative findings and the involvement of large vessels.

Keywords: Axilla, Cavernous hemangioma, Biopsy, Vascular neoplasms, Ultrasonography

\section{INTRODUCTION}

Axillary cavernous hemangiomas are extremely rare benign neoplasms, with only two cases reported in the English literature to date. In the first case, in 1982, a 22-year-old Caucasian woman presented with a large tender mass in the left axilla [1]. Although its exact size was not mentioned, the mass filled the axilla and the lateral thoracic surface of the long thoracic nerve, invading the hemisphere of the left breast. Owing to massive bleeding, surgery

Received: Nov 6, 2019 Revised: Dec 17, 2019 Accepted: Dec 27, 2019 Correspondence to: Min-Ki Seong

Department of Surgery, Korea Cancer Center Hospital, Korea Institute of Radiological and Medical Sciences, 75 Nowon-ro, Nowon-gu, Seoul

01812, Korea

Tel: +82-2-970-1251, Fax: +82-2-970-2419

E-mail: mklegend@kcch.re.kr

ORCID: Jihye Choi (https://orcid.org/0000-0002-6580-6115), Chan Sub Park (https://orcid.org/0000-0003-3039-8868), Joonseog Kong (https://orcid.org/00000003-4151-4617), Hyun-Ah Kim (https://orcid.org/0000-0001-9713-2605), Woo Chul Noh (https://orcid.org/0000-0003-3770-1612), Min-Ki Seong (https://orcid. org/0000-0002-9075-9365)

Copyright (C) 2019 Korean Society of Surgical Oncology

This is an Open Access article distributed under the terms of the Creative Commons Attribution Non-Commercial License (http://creativecommons.org/licenses/by-nc/4.0) which permits unrestricted non-commercial use, distribution, and reproduction in any medium, provided the original work is properly cited. was unsuccessful, and the remnant hemangioma was treated by using a carbon dioxide laser. In the second case, in 2008, a 43-year old Brazilian woman presented with an axillary mass and adjacent lymphadenomegaly, raising suspicion of a malignant breast neoplasm [2]. Core needle biopsy was contraindicated owing to the high vascularity of the mass and its proximity to the thoracic wall and axillary vessels. Excisional biopsy, however, was successful since the hemangioma was relatively small $(3 \mathrm{~cm})$. A salient point of the 2008 report was that axillary cavernous hemangiomas can mimic malignant cancers. Herein, we describe a surgically treated axillary cavernous hemangioma in a Korean woman and review the pertinent literature. The Institutional Review Board of the Korea Cancer Center Hospital approved the protocol version 1.0 (KIRAMS 2018-03-011).

\section{CASE REPORT}

A 30-year-old woman presented with an incidentally detected mass in the left axilla. She had no underlying diseases or self-reported history of surgery, trauma, or oral contraceptive use. Physical examination revealed a non-tender, easily movable mass in the left axilla (Fig. 1). Mammography images of the axilla and left breast were unremarkable (Fig. 2A-C). Ultrasonography revealed a 4.8-cm solid mass with a lobulated margin (Fig. 2D). Color Dop- 


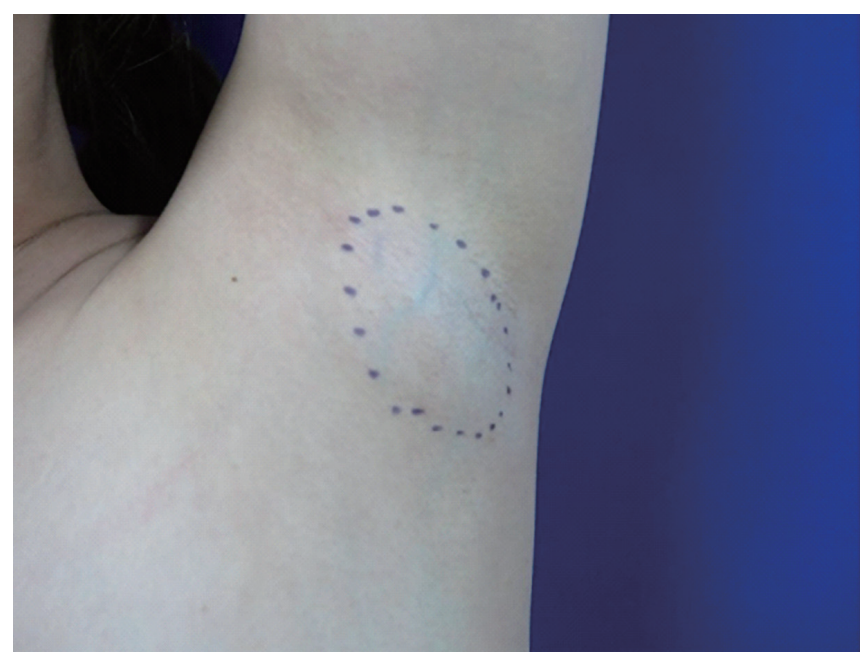

Fig. 1. A palpable mass located in the left axillary area at initial presentation. The mass was approximately $4 \mathrm{~cm}$ in size and ovalshaped (dotted circle). On palpation, it was easily movable, rather soft, and non-tender. Although it protruded somewhat, the surrounding skin appeared normal.

pler ultrasound detected minimal blood flow within the mass (Fig. $2 \mathrm{E})$. The mass was initially suspected to be either a lymphoma or phyllodes tumor based on the categories in Breast Imaging-Reporting and Data System IVb.

A core needle biopsy was performed without complications. Microscopically, the core needle biopsied specimen consisted of thin-walled, cystically dilated lumens without intra-luminal cellular components (Fig. 3A). Because whether it originated in a vessel or lymph node was unclear, immunohistochemical staining was performed. Immunohistochemical staining of CD34, a marker of the vascular endothelium, was positive (Fig. 3B) while staining of D2-40 (podoplanin), a marker of the lymphatic endothelium, was negative (Fig. 3C), resulting in an unexpected diagnosis of cavernous hemangioma.

Because this diagnosis differed from that of the radiological examinations, an excisional biopsy was performed for confirmatory purposes and to rule out malignancy. Infiltration of the mass into the axillary vein was observed intraoperatively; consequently, the vein was sacrificed after identifying the collateral vessels. Macroscopically, the excised mass measured $4.5 \mathrm{~cm}$, and the cut surface had multicystic spaces that exuded old blood-colored fluid (Fig. 4). Microscopically, the mass contained dilated, thin-walled vessels with red blood cells in the lumens (Fig. 3D), a distinct characteristic of a cavernous hemangioma. Cellular atypia was not observed (Fig. 3E). Based on these findings, the final pathological diagnosis was cavernous hemangioma.

Ulnar nerve palsy developed postoperatively, possibly due to an
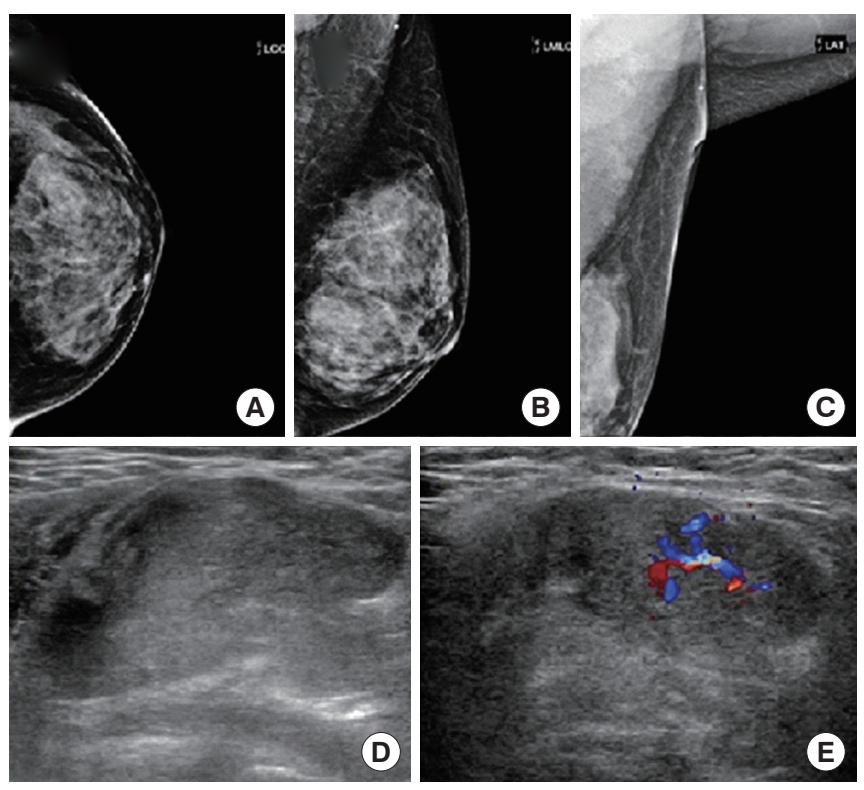

Fig. 2. Preoperative imaging findings for the axillary mass. (A-C) There were no remarkable findings on a mammogram: $(A)$ cranio-caudal view, (B) medio-lateral view, and (C) axillary tail view. (D) A transverse sonogram of the left axilla shows a gently lobulated, circumscribed hypoechoic nodule. (E) A color Doppler sonogram shows minimal blood flow within the mass.

indirect thermal injury or traction injury. The palsy resulted in a loss of sensation in the innervating area and an approximate 30\% reduction in grip strength. After rehabilitation and steroid therapy, grip strength recovered almost fully to the normal range, while the numbness did not recover. No evidence of recurrence was observed during a 23-month follow-up.

\section{DISCUSSION}

Cavernous hemangiomas are benign endothelial cell neoplasms. Although most common in the head and neck, they can occur anywhere in the body, and predominantly affect women 20 to 30 years of age. Historically, content type and channel size were used to distinguish cavernous hemangiomas, which often caused confusion. The newer classification system by Mulliken et al. [3] defines hemangiomas as endothelial malformations. Unlike vascular malformations, cavernous hemangiomas are absent at birth, proceed with tumor growth (due to endothelial cell proliferation) followed by tumor regression [4]. In adulthood, cavernous hemangiomas rarely regress spontaneously and require surgical removal when they exert pressure on surrounding structures, causing local destruction [5]. Exogenous estrogen use, as in hormone replacement therapy, for example, may promote the development of hemangiomas [6], although this was not a factor in our case. 

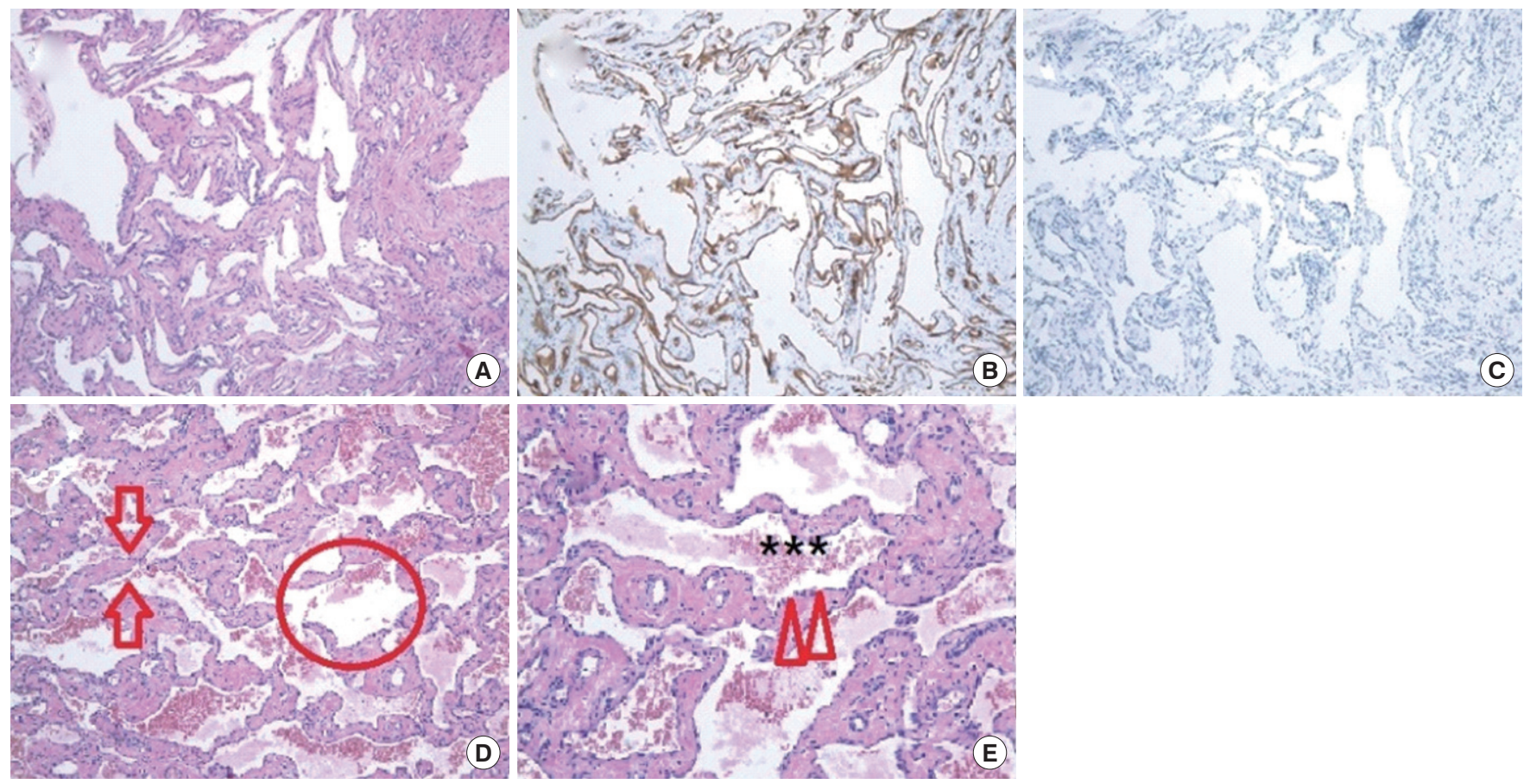

Fig. 3. Microscopic views of a core needle biopsy specimen $(A-C)$ and the excised mass $(D, E)$. (A) The specimen consisted of thin-walled, cystically dilated lumens without intra-luminal cellular components $\left(H \& E_{1} \times 100\right)$. Whether it originated in a vessel or lymph node was unclear. Consequently, immunohistochemical staining was performed. (B) Immunohistochemical staining of CD34, a marker of the vascular endothelium, was positive (x100). (C) Immunohistochemical staining of D2-40 (podoplanin), a marker of the lymphatic endothelium, was negative $(\times 100)$. Therefore, the diagnosis was cavernous hemangioma. (D) The excised mass was composed of cystically dilated lumina (circle) and thin-walled vessels (arrows) $\left(H \& E_{1} \times 100\right)$. (E) The cysts were lined with endothelial cells (arrowheads) and contained red blood cells (asterisks), a distinct characteristic of a cavernous hemangioma. Cellular atypia was not present $(H \& E, \times 200)$. Therefore, the final diagnosis was cavernous hemangioma. Immunohistochemical staining was not necessary in this setting.
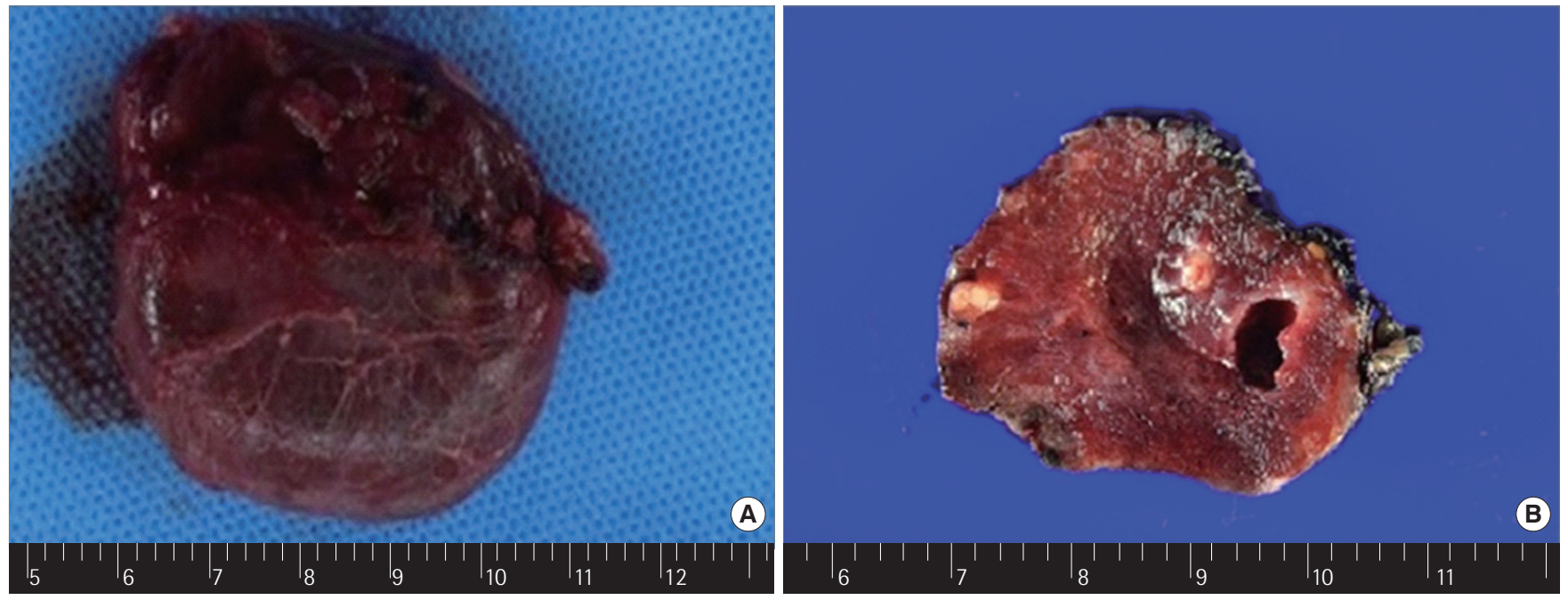

Fig. 4. Macroscopic views of the excised mass. (A) The excised mass was $4.5 \times 4.0 \times 1.7 \mathrm{~cm}$ in size and was partly solid and partly cystic. (B) The cut surface of the mass had cystic spaces exuding old blood-colored fluid. A scale ruler with 1-cm subdivisions is shown.

Hemangiomas should be clinically diagnosed with caution as they sometimes resemble malignant cancers, which differ in terms of prognosis and risk of recurrence. The differential diagnoses for hemangioma include lipoma, lymphoma, an epidermal cyst, a 
phyllodes tumor, well-differentiated angiosarcoma, and breast cancer originating in an accessory breast or axillary tail [2]. Unlike hemangiomas, angiosarcomas are often palpable, larger than $3 \mathrm{~cm}$, and ill-defined [6,7]. However, axillary hemangiomas can present with a large size $($ e.g., $4.5 \mathrm{~cm})$ and imprecise margins, as shown in the present report.

Cavernous hemangiomas are difficult to diagnose preoperatively via conventional imaging techniques as they lack pathognomonic characteristics. In some cases, including ours, they are invisible on mammograms [8]. On sonograms, hemangiomas often display variable echotextures and vascularity depending on their histological components, which complicates diagnosis. Vascularity on color Doppler sonograms is not required for diagnosis as blood flow may be restricted in the malformed vessels [9]. Magnetic resonance imaging (MRI) may be helpful in characterizing the lesions and identifying the anatomical extent of the disease, which is critical for treatment planning [4].

Diagnosing vascular tumors via core needle biopsy is challenging. To minimize potential contamination, the needle should not pass through the tumor, and the needle tract should lie within the coverage area of the pending surgery. In addition, injury to vital structures such as neurovascular bundles should be avoided. An excisional biopsy is usually only required when the preoperative core needle biopsy and radiological findings are discordant; in addition to confirming the diagnosis, excisional biopsies can also rule out malignancy, as in the present case $[10,11]$.

Treatment options for hemangiomas include surgical excision, percutaneous sclerosis, embolization, steroid injections, and radiation [12]. Asymptomatic hemangiomas can be simply observed, while deeply located hemangiomas should be treated via sclerosis, embolization, or radiotherapy. Surgical excision is preferred for small confined lesions. If completely excised, a cavernous hemangioma has a good prognosis. In cases of incomplete removal, additional wide excision may be necessary given the reported recurrence rate of $10 \%[13,14]$.

Despite the benign nature of hemangiomas, surgery was challenging in our case because of the involvement of large vessels. Moreover, it may have triggered the postoperative development of mild ulnar nerve palsy. Bellina [1] described a case of a large axillary cavernous hemangioma with imprecise margins in which complete surgical extirpation was unsuccessful. Kim et al. [15] found that hemangiomas in deeper body compartments tend to be invasive on presentation and larger than those in superficial compartments; individualized treatments for the former were recommended. Although complete excision with wide margins reduces the risk of recurrence, it increases the risk of injuries to vital neighboring structures. Therefore, when a hemangioma is suspect- ed, its treatment should be based on its depth of location, size, and margin status and the possibility of malignancy. When considering surgery, postoperative benefits versus potential complications should be carefully weighed.

In conclusion, we describe an extremely rare axillary cavernous hemangioma and its surgical outcome in a Korean woman. Even when an axillary mass presents with inconsistent preoperative biopsy and radiological findings, axillary cavernous hemangioma should not be ruled out despite its rarity. This is especially important in specialized breast clinics where the focus is usually on the diagnosis and treatment of malignant breast neoplasms. Particular attention should be paid during dissection to the anatomical location of the mass and its effect on neighboring structures. Detailed preoperative imaging procedures, including MRI, should be performed.

\section{CONFLICT OF INTEREST}

No potential conflict of interest relevant to this article was reported.

\section{ACKNOWLEDGMENTS}

This study conducted with the help of a grant from the Korea Institute of Radiological and Medical Science (KIRAMS), funded by the Ministry of Science and ICT (MSIT), Republic of Korea (No. 50472-2018).

\section{REFERENCES}

1. Bellina JH. Cavernous hemangioma of left axilla and breast: a new surgical approach with the carbon dioxide laser. J Reprod Med 1982;27:146-8.

2. da Silva BB, Pires CG, Pereira-Filho JD, Dos Santos AR, Lopes-Costa PV. Cavernous hemangioma in the axillary region mimicking malignant neoplasia. South Med J 2008;101:1051-3.

3. Mulliken JB, Fishman SJ, Burrows PE. Vascular anomalies. Curr Probl Surg 2000;37:517-84.

4. Donnelly LF, Adams DM, Bisset GS 3rd. Vascular malformations and hemangiomas: a practical approach in a multidisciplinary clinic. AJR Am J Roentgenol 2000;174:597-608.

5. Kim SJ, Han HS, Kim JS, Park JH, Jeon HJ, Yi JG. Cavernous hemangioma of the breast parenchyma with unusual features. J Ultrasound Med 2006;25:1343-6.

6. Mesurolle B, Wexler M, Halwani F, Aldis A, Veksler A, Kao E. Cavernous hemangioma of the breast: mammographic and sonographic findings and follow-up in a patient receiving hormone-replacement therapy. J Clin Ultrasound 2003;31:430-6. 
7. Glazebrook KN, Morton MJ, Reynolds C. Vascular tumors of the breast: mammographic, sonographic, and MRI appearances. AJR Am J Roentgenol 2005;184:331-8.

8. Gembala RB, Hayward CZ, Ball DS, Radecki PD, Hartman GG. Color Doppler detection of a breast perilobular hemangioma. J Ultrasound Med 1993;12:220-2.

9. Mesurolle B, Sygal V, Lalonde L, Lisbona A, Dufresne MP, Gagnon $\mathrm{JH}$, et al. Sonographic and mammographic appearances of breast hemangioma. AJR Am J Roentgenol 2008;191:W17-22.

10. Okada K. Points to notice during the diagnosis of soft tissue tumors according to the "Clinical Practice Guideline on the Diagnosis and Treatment of Soft Tissue Tumors". J Orthop Sci 2016;21:705-12.

11. Sebastiano C, Gennaro L, Brogi E, Morris E, Bowser ZL, Antonescu $\mathrm{CR}$, et al. Benign vascular lesions of the breast diagnosed by core needle biopsy do not require excision. Histopathology 2017;71: 795-804.

12. Lee SH, Shin KH, Yang WI, Suh JS, Hahn SB. Surgical treatment of hemangioma in the extremities. J Korean Orthop Assoc 2004; 39:215-21.

13. Bhathal PS, Brown RW, Lesueur GC, Russell IS. Frequency of benign and malignant breast lesions in 207 consecutive autopsies in Australian women. Br J Cancer 1985;51:271-8.

14. Allen PW, Enzinger FM. Hemangioma of skeletal muscle: an analysis of 89 cases. Cancer 1972;29:8-22.

15. Kim YS, Choi HL, Lee JM, Lee HS, Kim JR. Analysis of the recurrence after surgical treatment of the hemangioma in the extremities. J Korean Bone Joint Tumor Soc 2010;16:74-9. 